\title{
O que é Emoção? Em Busca da Organização Estrutural do Conceito de Emoção em Crianças
}

\author{
What is Emotion? Searching the Organizational Structure \\ of Children's Concept of Emotion
}

\author{
Antonio Roazzi*, a , Maria da Graça Bompastor Borges Diass ${ }^{\mathrm{a}}$ Janaína Oliveira da Silva ${ }^{\mathrm{b}}$, \\ Luciana Barboza dos Santos ${ }^{\mathrm{c}}$, \& Maíra Monteiro Roazzi ${ }^{\mathrm{d}}$ \\ ${ }^{a}$ Universidade Federal de Pernambuco, Recife, Brasil baculdade da Serra Gaúcha, Caxias do Sul, Brasil

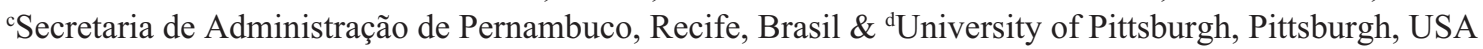

Resumo

A realização do presente estudo teve como objetivo principal investigar a organização estrutural do campo semântico conceitual da emoção em crianças em uma perspectiva êmica. Participaram desta pesquisa 247 crianças de escola pública e particular, com idades entre sete e 12 anos. Foram aplicadas as técnicas de associação livre de palavras (emoção) e duas perguntas (freqüência das emoções e o que sabiam sobre emoção). Essa ultima questão apontou que a maioria das crianças respondia relacionando emoções a sentimento, a coisas que sentem no coração. Elas também traziam exemplos de acontecimentos positivos e negativos, citavam emoções específicas, principalmente as classificadas como primária, pelos teóricos da área. Na questão relativa à freqüência destas emoções em sua vida as crianças apontaram alegria, felicidade e tristeza como as emoções mais vivenciadas no seu dia a dia. Na associação livre, as emoções primárias apareceram de forma predominante, destacando-se em primeiro lugar a alegria, seguida da tristeza, felicidade, raiva, medo e amor respectivamente. Emoções mais elaboradas, consideradas secundárias, também foram encontradas como saudade, ódio, paz, ansiedade, etc. Para verificar a organização estrutural do campo semântico conceitual, as categorias produzidas através da associação livre foram analisadas também por meio da Análise da Estrutura de Similaridade (SSA). Em seguida, para verificar a relação entre a estrutura decorrente do campo semântico encontrado e as variáveis idade, sexo, e tipo de escola, os dados foram analisados através do método das "variáveis externas como pontos". Regressões múltiplas foram também computadas. Foi encontrado que o tipo de escola e, sobretudo, a idade desempenharam um papel relevante em influenciar de forma significativa a organização estrutural do campo semântico conceitual das emoções, que apresentou uma estrutura ordenada de tipo modular.

Palavras-chave: Emoção; Sentimentos; Conceito; Representações sociais.

\section{Abstract}

The main aim of this study was to investigate the structural organization of the semantic concept of emotion in children based on an emic perspective. A free word association (emotion) and two questions (frequency of emotions and what they knew about emotions) was applied to a sample of 247 children aged 7-12 from public and private schools in Brazil. The question concerning what they knew about emotions indicated that most children link emotions to feelings, things their heart feels. Participants also gave examples of positive and negative experiences, specifying certain emotions, mainly emotions classified as primary by researchers in this field. Regarding the question_about the frequency of those emotions in their lives, they pointed out joy, happiness and sadness as being the emotions frequently experienced on their daily lives. On the free word association primary emotions predominated being joy the most frequent one, followed by sadness, happiness, anger, fear and love. We also encountered more elaborate emotions (secondary emotions) such as missing someone, hatred, peace, and anxiety, among others. In order to verify the structural organization of their semantic conceptual field, we analyzed the categories that participants emitted on the free association task by means of Structural Similarity Analysis (SSA). A method of "external variables as points" was applied with the intention of verifying the relation between the structure created from the semantic field and the variables of age, sex and type of school. Multiple regressions were also computed. It was found that the type of school and, especially, age played a relevant role in predicting the structural organization of emotions, i.e. an ordered modular structure.

Keywords: Emotion; Feeling; Concept; Social representations.

A emoção é um fenômeno complexo, um processo que envolve todo o organismo. Tal complexidade produz di-

"Endereço para correspondência: Universidade Federal de Pernambuco, Centro de Ciências Humanas, Rua Acadêmico Hélio Ramos, s/n, Cidade Universi-tária,
Recife, PE, Brasil, CEP 50670-901. E-mails: roazzi@ gmail.com e mgbbdias@yahoo.com.br

${ }^{1}$ Ekman e Friesen $(1971,1978)$, ao replicarem vários 
ficuldades em seu estudo, a primeira diz respeito a sua própria definição e a segunda ao compartilhamento do seu significado. De fato, à primeira vista há um entendimento entre as pessoas do que seja emoção, no entanto, ao ter que defini-la percebe-se que esta não é uma tarefa fácil. Além disso, embora várias pessoas utilizem a mesma palavra numa tentativa de definição, não significa que o entendimento seja o mesmo entre elas. Como conse-qüência destas dificuldades o conhecimento das emoções é ainda parcial e fragmentado, e muitas são as questões que se encontram ainda em aberto (e.g., Davidson, Scherer, \& Goldsmith, 2003; Ekman \& Davidson, 1994; Lazarus, 1991; Levenson, 2001). Uma destas refere-se à conceitualização das mesmas ao longo do desenvolvi-mento ontogenético. Pesquisas recentes (e.g., Barrett, 2006a, 2006b; Davidoff, 2001; Davies \& Davidoff, 2000; Lindquist, Barrett, Bliss-Moreau, \& Russel, 2006; Roberson \& Davidoff, 2000; Russell, 1997; Russell \& Widen, 2002; Zammuner, 1998, 2001) sugerem um modelo teórico de base para conceitualizar o conhecimento e as representações que as pessoas elaboram sobre as emoções a partir de categorias de objetos naturais ou eventos. Estas categorias são formadas como um resul-tado de experiências repetidas e se tornam organizadas como protótipos (Rosch, 1975; 1983; Rosch \& Lloyd, 1978; Rosch, Varela, \& Thompson, 1991). O conjunto inter-relacionado de categorias de emoções organiza-se e estrutura-se de acordo com uma dimensão abstrato-concreto, de forma hierárquica. Na base desta hierarquia encontra-se um conjunto de emoções como amor, feli-cidade, raiva, tristeza, medo e surpresa, extremamente útil para poder fazer distinções no dia a dia. Estas são basicamente as palavras que as pessoas costumam usar ao serem perguntadas sobre as emoções (Fehr \& Russell, 1984), são as que as crianças aprendem a falar primeiro (Bretherton \& Beeghly, 1982; Bretherton, Fritz, Zahn-Waxler, \& Ridgeway, 1986) e o que os teóricos nesta área têm definido como emoções básicas ou primárias (idéia iniciada por Darwin, 1872/1975) $)^{1}$. Apesar dos inúmeros estudos sobre estas emoções básicas ou primárias a respeito de sua hierarquia, não têm sido realizados estudos investigando como esta organização estrutural se forma ao longo do desenvolvimento. De fato, os poucos estudos sobre a organização estrutural das emoções têm sido geralmente realizados com populações adultas (e.g. Albelson \& Sermat, 1962; Canter \& Ioannou, 2004; Fisher, Heise, Borhnstedt, \& Lucke, 1985; Plutchik, 1962; Russell, 1979; Schlosberg, 1954; Watson \& Tellegen, 1985).

A realização do presente estudo teve como objetivo prin-

estudos de Darwin (1872/1975) sobre as expressões faciais através do FACS (Facial Action Coding System: sistema de codificação das expressões faciais), chegaram a um padrão ou protótipo morfológico de cada emoção. Os estudos de Ekman e Friesen incluem investigações com diferentes culturas e com outros elementos da reação emocional e seus trabalhos, assim como os de Darwin dizem respeito à emoções básicas como alegria, tristeza, raiva, nojo, surpresa, medo.

${ }^{2}$ Sobretudo a $3^{\mathrm{a}}$, $4^{\mathrm{a}}$ e $5^{\mathrm{a}}$ parte da Ethica dedicadas ao cipal investigar a organização estrutural do campo semântico conceitual da emoção em crianças em uma perspectiva êmica, visando melhor compreender a re-presentação da mesma (para maiores detalhes sobre organização estrutural do campo semântico-conceitual ver Roazzi, 1987; Roazzi \& Dias, 2001). A sua elaboração e execução, baseou-se na teoria das representações sociais e em estudos antropológicos que usam análises multidi-mensionais, tomando como ponto de partida os métodos e técnicas da antropologia cognitiva (D’Andrade, 1995; Lave, 1988).

\section{O que é Emoção?}

"É difícil dizer... Emoção é alguma coisa que a gente sente quando tá sentindo alguma coisa, né"? (11anos - sexo masculino/ Escola Particular).

Como sugere a resposta dessa criança, é difícil para um leigo dizer uma definição do que seja emoção, pois conforme sugerem vários teóricos da área (e.g, Lazarus, 1991; Levenson, 2001; Plutchik \& Conte, 1997), trata-se de uma dimensão complexa e multifacetada. Filósofos como Espinosa $(1677 / 1989)^{2}$ tentaram encontrar uma resposta convincente e até hoje cientistas também a buscam em suas pesquisas sem chegar a um consenso geral. Alguns aspectos que podem justificar esta dificuldade conceitual são: as formas de expressão das emoções modificam-se no decorrer da vida, ou seja, determinados estados emocionais tornam-se mais sofisticados à medida que o in-divíduo avança no seu processo de desenvolvimento; a existência de diferentes contextos sócio-culturais e do momento histórico, no qual o sujeito está inserido (Roazzi, Federicci, \& Wilson, 2001). A significação que cada sujeito atribui a suas emoções e o modo como elas são vivenciadas também deve ser um aspecto a ser consi-derado (Bruner, 1997). Deste modo, a presença destes aspectos contribui para emergência de perspectivas teóricas diversas (e.g., Averill, 1980; Damásio, 1999; Ekman, 1984; Izard, 1991; Mandler, 1975; Plutchik, 1994; Plutchik \& Kellerman, 1980).

Na presente investigação, buscaremos conceitos sobre a emoção, tendo em vista a importância que a produção de significados desempenha na ação humana (Bruner, 1997). Também será analisada a forma como os conceitos se organizam e se estruturam dentro de uma pers-pectiva de desenvolvimento, daí o nosso interesse par-ticular de uma investigação com crianças. Com a fina-lidade de alcançar o objetivo proposto, pensou-se em quatro perguntas centrais que nortearam esse estudo: Qual a organização estrutural do campo semântico con-ceitual das emoções em crianças? Qual a relação entre a estrutura decorrente do campo semântico encontrado e as variáveis idade, sexo, e tipo de escola? Quais as emoções são experienciadas com mais freqüência pelas crianças? Por último, como as crianças conceituam as emoções?

estudo dos "afetos".

${ }^{3} \mathrm{O}$ fato de não verificarmos as emoções de nojo encontra 
O conhecimento da emoção desenvolvido pelas crianças pode ser compreendido dentro do campo das representações sociais. Primeiramente, isto se deve ao fato desse conhecimento ser elaborado e partilhado socialmente com um objetivo prático pertencente a uma realidade comum (Jodelet, 1994). Em segundo lugar, envolve aspectos cognitivo, avaliativo, afetivo e simbólico (Wagner, 1998) e é produto e processo de uma atividade mental onde se reconstrói o real e se atribui uma significação particular (Abric, 1994). A importância das emoções nas representações sociais é ampliada por Moscovici (2000) quando ele afirma que todas as nossas experiências afeti-vas expressas em condutas, respostas corporais e verbais são conseqüência não de uma excitação exterior, mas sim da representação que construímos dela. Além disso, as emoções são indispensáveis para mobilizar as pessoas, para criar vínculos e representar o futuro:

... las representaciones sociales se han preocupa-do siempre de los aspectos de la sensibilidad social, sentimientos sociales, entre otros... . las represen-taciones sociales son indispensables para movilizar a la gente, para permitirse representarse el futuro $y$, también, para crear vínculos, puesto que hay algo puesto en común en el pensamiento, en los sentimien-tos, en el intercambio conversacional (Moscovici, 2000, p. 302-303).

Para embasar essa pesquisa, além das representações sociais, recorremos também a duas outras importantes perspectivas teóricas: a classificação das emoções proposta por Damásio (2004) e Harris (1996) e a mediação semiótica dos processos afetivos desenvolvida por Valsiner (2001). Damásio (2004) classifica as emoções em três categorias: emoções de fundo, primárias e so-ciais. As emoções de fundo são aquelas em que o sujeito tem a capacidade de decodificá-las rapidamente em diferentes contextos, sendo elas agradáveis ou desagradáveis. As emoções primárias ou universais são facilmente identificáveis entre seres de uma mesma espécie, como, por exemplo, raiva, tristeza, medo, zanga, nojo, surpresa, felicidade. E finalmente, as emoções sociais ou secundárias que, de acordo com Damásio, são influenciadas pela sociedade e cultura, como a vergonha, o ciúme, a culpa, compaixão, embaraço, simpatia, orgulho (ver também apresentando evidências nesta perspectiva: Eid \& Diener, 2001; Harris, 1996; Markus \& Kitayama, 1991; Vikan \& Dias, 1996; Vikan, Roazzi, \& Dias, 2009).

Harris (1996) diferencia as emoções entre simples e complexas pelo fato de existir uma expressão facial reconhecível ou não. As emoções de raiva, medo, tristeza e alegria teriam expressões faciais mais facilmente reconhecíveis, sendo consideradas emoções simples. Crianças a partir de quatro ou cinco anos seriam capazes de indicar situações apropriadas para essas emoções. Já as emoções complexas não teriam uma figura facial ou expressões comportamentais tão óbvias como vergonha, orgulho e culpa. E apenas aos sete anos as crianças começariam a identificar estas emoções. Nessa idade a criança percebe que as pessoas em sua volta são afeta-das emocionalmente por eventos externos e não somente pelas conseqüências de suas ações. Deste modo, o seu campo de visão é ampliado e a aprovação ou desaprovação social passam a ser mais consideradas.

Ainda segundo Harris (1996), tanto emoções simples como emoções complexas podem ser positivas, nega-tivas ou mistas. As emoções positivas seriam oriundas de situações agradáveis; as negativas oriundas de situações desagradáveis. Com o passar do tempo, a criança aprende que determinadas situações de sua vida podem pro-vocar o surgimento de emoções positivas e negativas ao mesmo tempo. Esta diversidade de emoções que abrange sentimentos de ambivalência relacionados a uma única situação é chamada de emoções mistas. Por exemplo: Quando uma criança ganha de presente uma bicicleta que tanto desejava, fica feliz (emoção positiva), mas ao mesmo tempo, o fato dela ainda não saber andar de bicicleta a faz sentir medo (emoção negativa) de cair.

Valsiner (2001) em sua perspectiva teórica da mediação semiótica dos processos afetivos propõe um desenvolvimento ontogenético em três níveis: (a) nível pré-lingüístico (subdividido em nível 0 , referente ao estado inato de excitação, e em nível 1, referente as emoções corporais, a tonalidade emocional imediata); (b) nível linguístico (subdividido no nível 3, quando categorias específicas de emoções são nomeadas, e nível 4, quando há ações discursivas complexas); e (c) nível paralin-güístico (quando há estados afetivos complexos não expressáveis por palavras).

$\mathrm{Na}$ literatura acerca das emoções em crianças, foram encontradas pesquisas investigando a habilidade das crianças reconhecerem expressões faciais das emoções (e.g., Caron, Caron, \& Myers, 1982; Rieffe, Terwogt, \& Stockmann, 2000); a habilidade das crianças em responderem apropriadamente descrições verbais de situações que recrutam emoções (Camras \& Allison, 1985); e a consciência das crianças sobre as causas e consequências das emoções (Manstead, 1993). Mas como as crianças conceituam as emoções ao longo do processo de desenvolvimento? Como esta conceitualização das emoções se estrutura ao longo do desenvolvimento? Isso nos levou a busca da organização estrutural do campo semântico conceitual da emoção em crianças.

$\mathrm{Na}$ literatura sobre emoções em crianças não tem sido encontrados registros de estudos comparativos entre crianças de escolas publica e particular, apesar da existência de inúmeros estudos apontando significativas diferenças em tarefas que avaliam processos cognitivos (Roazzi, 1987) e no desempenho escolar (Demo, 2007). Só, recentemente Roazzi, Dias, Minervino, Roazzi e Pons (2009), em uma investigação visando avaliar em crianças de 4 aos 12 anos de escola publica e particular a compreensão das emoções encontraram diferenças bastantes significativas, o que nos levaram em considerar nesta investigação também esta variável.

Método 
Participantes

Participaram do estudo 247 crianças (129 meninos e 118 meninas), alunos de escola pública (escolas esta-duais) e particular (117 e 130 respectivamente) com idades entre sete e 12 anos. Maiores detalhes da amostra encontram-se na Tabela 1. Todos os alunos frequentavam escolas localizadas na região metropolitana de Recife. Enquanto os alunos de escola pública estudavam em três escolas estaduais, os alunos de escola particular estudavam em escolas privadas que atende um segmento da sociedade de classe média alta.

Tabela 1

Média da Idade em Meses de Acordo com Escola e Idade

$\begin{array}{lll}\text { Idade } & \text { Escola Pública } & \text { Escola } \\ \text { Particular } & \end{array}$

$\begin{array}{lrrrr} & & & & \\ & \text { Média } & \text { DP } & \text { Média } & \text { DP } \\ 7 & 88,60 & 6,76 & 88,93 & 4,21 \\ 8 & 102,64 & 3,54 & 101,10 & 2,40 \\ 9 & 115,11 & 3,44 & 114,68 & 3,91 \\ 10 & 125,13 & 3,50 & 127,23 & 3,71 \\ 11 & 139,32 & 3,61 & 135,45 & 3,12 \\ 12 & 153,22 & 4,22 & 152,70 & 3,01\end{array}$

\section{Procedimento}

Foram aplicadas as técnicas de associação livre e duas perguntas. Em primeiro lugar, através da técnica de associação livre, como meio de acesso ao campo semântico das representações, pedia-se que os sujeitos falassem livremente o que pensavam eom a evoeação da expressão "emoção": "Diga para mim algumas emoções que vem na sua cabeça?". Em seguida, foi também perguntada a freqüência destas emoções em sua vida: "Quais destas emoções você experiencia com mais freqüência"? Por último, através de uma entrevista, foi examinado o conhecimento que os participantes tinham das emoções através de uma pergunta direta. "Você sabe o que é uma emoção? Explique, por favor".

\section{Análise dos Dados}

Aplicou-se sobre as categorias produzidas uma Aná-lise da Estrutura de Similaridade (SSA, que é uma sigla das iniciais das palavras em inglês que significa Structural Similarity Analysis) juntamente com o método das "va-riáveis externas como pontos" (Roazzi \& Dias, 2001), para se estabelecer as correlações entre a estrutura decorrente do campo semântico encontrado e idade, sexo e tipo de escola. Todas as emoções produzidas através da associação livre com Coeficiente de Uniformidade da Distribuição igual ou superior a 5 foram plotadas numa região espacial através de uma análise SSA com as variáveis externas sexo, idade e tipo de escola (variáveis tipo "dummy”) (para maiores detalhes ver Roazzi \& Dias, 2001). Este modo de avaliação possibilitou que as respostas fossem localizadas espacial- mente, de modo que quanto mais próximas entre si, mais similaridades entre as emoções produzidas. No entanto, esse tipo de análise tornou-se inviável para a terceira questão, devido ao grande número de respostas diversificadas que demandaram um quantitativo de categorias além do indicado para análise correspondente.

Além das análises multidimensionais, para verificar os efeitos preditores das variáveis idade, sexo e tipo de escola nas emoções produzidas através da associação livre, das emoções experimentadas com maior freqüência e das categorizações na tarefa de definição de emoção foram computadas uma série de análises de regressões lineares tipo passo-a-passo (stepwise).

\section{Resultados e Discussão}

\section{1 a Questão - Associação Livre Sobre as Emoções}

A partir da associação livre de palavras à pergunta "Diga para mim algumas emoções que vem na sua cabeça?”, obteve-se 32 tipos de expressões semânticas (em média cada crianças produzia três palavras), as quais, ao serem agrupadas de acordo com a similaridade de significados, originaram as categorias apresentadas na Tabela 2. As emoções por ordem de maior ocorrência foram: alegria (128 vezes); tristeza (115 vezes); felicidade (73); raiva (51); medo (43); amor (38); saudade (25); susto (12); ódio (10), chateado e lágrimas (7), carinho e paz (6) e angústia (5). Todos estes itens apresentavam um Coeficiente de Uniformidade da Distribuição igual ou acima de 5 (considerados adequado para análises Multidimensionais não- paramétricas; para mais detalhes sobre este índice ver Amar \& Toledano, 2002). Outros itens produzidos com baixa freqüência e Coeficiente de Uniformidade da Distribuição abaixo de 5 foram: paixão, solidão, ansiedade, amizade, dor, sentimento, vergonha, contente, arrependimento, compaixão, bondade, saúde, sonhar, grito, desespero, desejo, inveja, sofrimento e falta ${ }^{3}$. Este fato causa grande surpresa visto que o nojo é tido como uma emoção primária (Damásio, 2004) o que nos leva a questionar se realmente ela seria rapidamente identificada nas mais diversas culturas e se, portanto, talvez não devesse se enquadrar nessa categoria.

Na Tabela 2 estão dispostas as freqüências, percentagens e coeficiente de distribuição de cada emoção.

\section{Tabela 2}

Freqüência, Coeficiente de Uniformidade da Distribuição e \% das Associações mais Freqüentes Produzidas a partir da Tarefa de Associação Livre "Diga para Mim Algumas Emoções que Vem na Sua Cabeça?” 
Roazzi, A., Dias, M. G. B. B., Silvia, J. O., Santos, L. B. \& Roazzi, M. M. (2011). O que é Emoção? Em Busca da Organização Estrutural do Conceito de Emoção em Crianças.

$\begin{array}{lrcc}\text { Associação Freqüência } & \begin{array}{c}\% \text { das emoções } \\ \text { mais freqüentes } \\ (\mathrm{N}=526)\end{array} & \begin{array}{c}\text { Coeficiente } \\ \text { de Uniformidade } \\ \text { da Distribuição }\end{array} \\ \text { Alegria } & 128 & 24,33 & 99 \\ \text { Tristeza } & 115 & 21,86 & 92 \\ \text { Felicidade } & 73 & 13,88 & 62 \\ \text { Raiva } & 51 & 9,69 & 44 \\ \text { Medo } & 43 & 8,17 & 37 \\ \text { Amor } & 38 & 7,22 & 33 \\ \text { Saudade } & 25 & 4,75 & 21 \\ \text { Susto } & 12 & 2,28 & 10 \\ \text { Ódio } & 10 & 1,90 & 9 \\ \text { Chateado } & 7 & 1,33 & 7 \\ \text { Lagrimas } & 7 & 1,33 & 6 \\ \text { Carinho } & 6 & 1,14 & 6 \\ \text { Paz } & 6 & 1,14 & 5 \\ \text { Angústia } & 5 & 0,95 & \end{array}$

Um problema que se apresenta quando se tenta classificar as emoções diz respeito às similitudes de vocabulário próprios da língua portuguesa, como é o caso de alegria e felicidade, raiva e ódio, susto e surpresa, as quais optamos por não agrupar para lançarmos mão de possíveis reflexões. Como por exemplo, será que emoções semelhantes possuem a mesma representação?

Do ponto de vista estrutural a partir da análise SSA, o campo semântico nessa tarefa de associação livre configurou-se numa estrutura modular, ou seja, havendo uma concentração de emoções primárias na região central e de emoções secundárias na região periférica (ver Figura 1). Este tipo de configuração, além do fato de indicar que existe uma ordem na estrutura das emoções, indica que as emoções primárias - que apresentam uma maior freqüência - estão mais relacionadas entre si, apresentam um caráter mais geral do que as secundárias e são mais facilmente compartilhadas pelas crianças, provavelmente pelo fato de serem mais facilmente lembradas diante da palavra-estímulo "emoção". As emoções secundárias pelo fato de estarem localizadas na periferia da estrutura modular estariam relacionadas a aspectos mais específicos em função da baixa correlação entre os mesmos. Estas características dos dois grupos de emoções encontram respaldo na literatura (Damásio, 2004; Harris, 1996; Vikan \& Dias, 1996).

Foram computadas uma série de regressões Stepwise considerando como variáveis dependentes as emoções produzidas através da associação livre e como variáveis independentes idade, sexo e tipo de escola. A variável idade apresentou efeitos preditores significantes para as emoções felicidade, amor e tristeza (explicando 1,7\% $\left[\mathrm{F}_{\text {change }}(1,230) 3,90, \mathrm{p}<0,049\right], 11,6 \%\left[\mathrm{~F}_{\text {change }}(1,230)\right.$ $30,09, p<0,001]$ e $2,4 \%\left[\mathrm{~F}_{\text {change }}(1,229) 5,754, \mathrm{p}<0,017\right]$ da variância, respectivamente). A variável tipo de escola apresentou um efeito preditor somente para tristeza

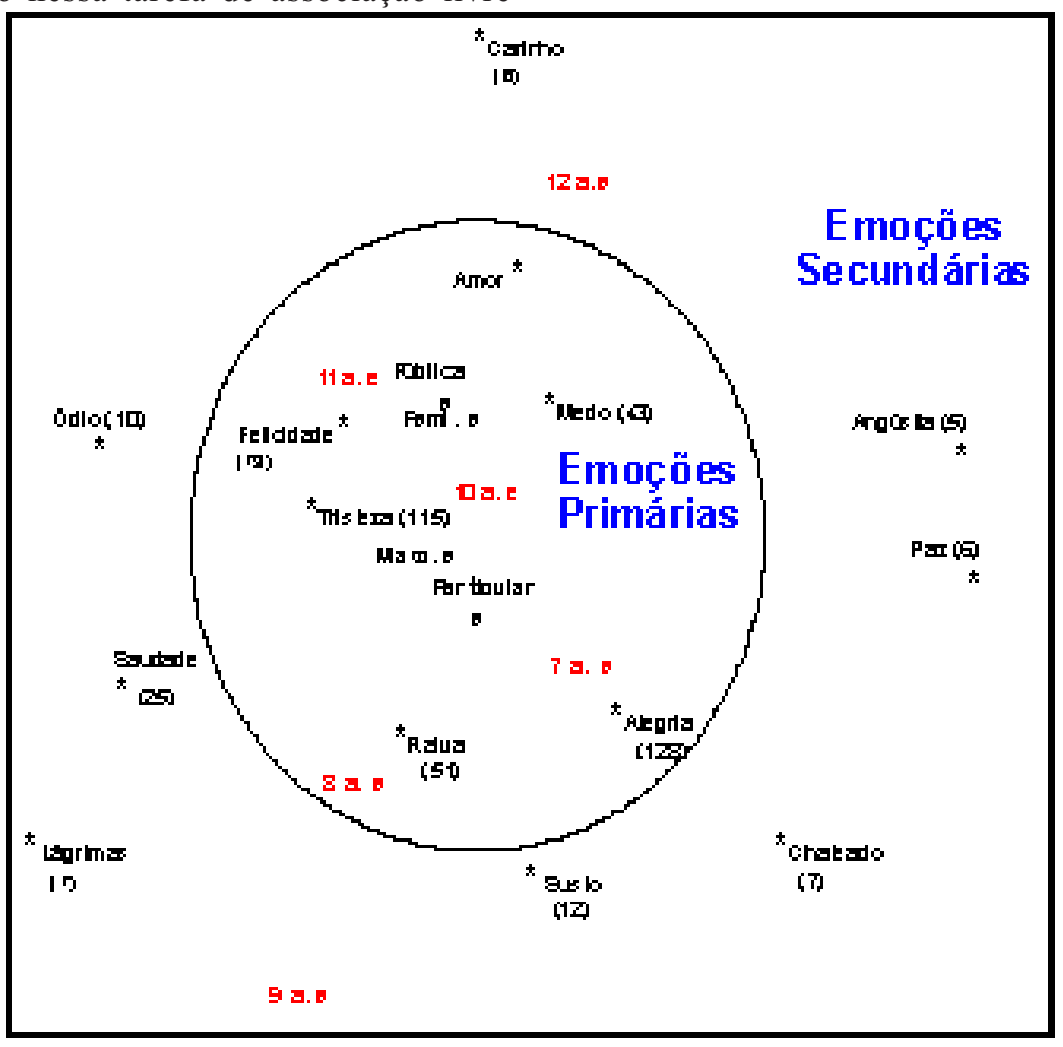

Figura 1. Análise SSA das emoções produzidas na Associação Livre considerando como variáveis externas (e) o tipo de escola, o sexo e a faixa etária.

Nota. Projeção tridimensional, Coordenada 1 x 2, Coeficiente de Alienação 0,12. Freqüência (em parêntese). 
$2,5 \%\left[\mathrm{~F}_{\text {change }}(1,230) 5,998, \mathrm{p}<0,015\right]$. Sexo não apresentou efeitos preditores significantes para nenhuma emoção. Esta falta de diferença pode ser observada também na Figura 1 pela proximidade existente entre o grupo do sexo feminino e o grupo do sexo masculino. Esse resultado levou-nos a considerar que crianças do sexo mas-culino e feminino, no que diz respeito às emoções, compartilham o mesmo campo semântico.

$\mathrm{O}$ efeito preditor nas emoções de tristeza, felicidade e amor da variável idade indica que quanto maior a idade, maior o surgimento dessas emoções, sobretudo a última 'Amor'. Uma possível explicação para tal constatação refere-se ao fato dessas crianças estarem iniciando a fase da pré-adolescência, momento em que as aquisições de um pensamento lógico formal as levam a questionar e considerar as adversidades do mundo, de si próprio e dos outros. Os adolescentes costumam vivenciar as emoções de forma mais intensa, experienciando sentimentos con-traditórios com maior freqüência, e passam a utilizar terminologias mais sofisticadas para representar suas emoções, como é o caso do termo felicidade ao invés de alegria. E, ainda, o despertar do interesse em estabelecer relacionamentos amorosos pode está vinculado ao sur-gimento em maior freqüência da emoção "amor" nessas crianças maiores.

Podemos também refletir sobre esse fenômeno a partir do paradigma histórico-cultural. Ao propor uma abordagem unificadora do desenvolvimento humano, Vygotsky defende o desenvolvimento de um sistema de conceitos. Os diferentes tipos de conceitos vão surgindo durante o desenvolvimento ontogenético, possibilitando a mudança (transição) de conceitos situacionais para um modo mais abstrato de conceito. A partir do momento que a criança tem acesso à escolarização e ao desenvolvimento científico, ela começa a pensar sobre a natureza dos próprios conceitos, ocorrendo uma mudança qualitativa re-lacionada aos processos metacognitivos. Dentro desta perspectiva, os aspectos cognitivos e afetivos são con-ceitualizados como fazendo parte de um todo complexo de inter-relações que são mais bem compreendidos pela criança mais tardiamente, por volta dos nove anos de idade (Oliveira \& Oliveira, 1999).

Quanto ao efeito preditor da variável 'tipo de escola' em relação à emoção de tristeza, é importante ressaltar que esta emoção teve menor frequiência nas crianças de escola pública em comparação com as crianças de escola particular. Tal resultado parece contradizer algumas expectativas do senso comum que na escola pública, onde se acolhe crianças com dificuldades socioeconômicas a emoção "tristeza" estaria mais propícia a ter uma re-presentatividade maior entre as crianças. Entretanto, isto não significa dizer que a alegria esteja mais presente na representação social das crianças de escola pública, apenas que o fator socioeconômico não é um preditor na emoção de tristeza dessas crianças.

$2^{\mathrm{a}}$ Questão - Freqüência das Emoções

A partir da análise SSA (Figura 2) das emoções podemos constatar que as crianças menores, com idades entre sete e oito anos, produziram de forma mais significativa respostas relacionadas com a categoria "não sabe". As crianças maiores (nove a 12 anos) ao definir as emoções conseguiram elaborar conceitos positivos (em maior freqüência) e negativos (numa freqüência menor). Ao falarem acerca das emoções sentidas com maior freqüência, as crianças não mencionam as emoções mistas, ou seja, elas falam de si mesmas utilizando emoções positivas ou negativas. As emoções mistas são destacadas no momento em que falam sobre as emoções de um modo geral, sem se remeter à experiência particular.

$\mathrm{Na}$ análise de regressão, constatou-se que Alegria é referida como sendo a emoção vivenciada com maior freqüência pelas crianças de escola particular - o tipo de escola explicando $2,0 \%$ da variância $\left(\mathrm{F}_{\text {change }}(1,244)\right.$ $4,867, \mathrm{p}<0,028)$. Esse resultado pode confirmar uma idéia comum de que, sendo o tipo de escola um indicador de nível socioeconômico, a escola pública, que se refere a um nível baixo, estaria mais propensa a privações e situações desencadeadoras de emoções desagradáveis. Enquanto a escola particular indicadora de um nível socio-econômico mais elevado favoreceria um ambiente de mais cuidado e protegido, aparentemente facilitando o surgimento maior de emoções mais agradáveis.

Este resultado aparentemente entra em contradição com o resultado da primeira tarefa, pois a tristeza aparece mais associada à escola particular. No entanto, convém salientar que na questão de associação livre a criança é convidada a falar sobre a emoção de um modo geral, enquanto nessa segunda questão ela é convidada a falar dela própria, da sua realidade específica. Em outras palavras, a criança de escola particular tem uma representação das emoções mais relacionada à tristeza, mas quando ela fala da própria emoção ela associa com alegria. Isso não significa dizer que as crianças de escola pública têm uma representação inversa das emoções, pois elas parecem se posicionar mais de forma neutra.

A partir desta constatação, podemos fazer menção ao teórico Henry Wallon (1941/1981), segundo o qual o desenvolvimento infantil é marcado por regressões, con-flitos e contradições que propiciam a reformulação e ampliação de conceitos e funções. A mudança de cada estágio representa uma evolução mental qualitativa por caracterizar um tipo diferenciado de comportamento que será substituída no estágio seguinte, o qual irá atribuir novas formas de pensamento, de interação social e de emoções direcionados ora para a construção do próprio sujeito, ora para a construção da realidade exterior. No nosso caso, ao responder a primeira questão da pesquisa, a criança dirigiu seus pensamentos e emoções para a realidade exterior ao refletir sobre as emoções enquanto representação social. Por outro lado, na segunda questão, ela se voltou para aspectos relacionados à construção de si mesma, pois se remetia à experiência pessoal para uma análise da freqüência das próprias emoções.

Outro resultado encontrado foi em relação à categoria (variável dependente) "não sabe": Quanto maior a criança 


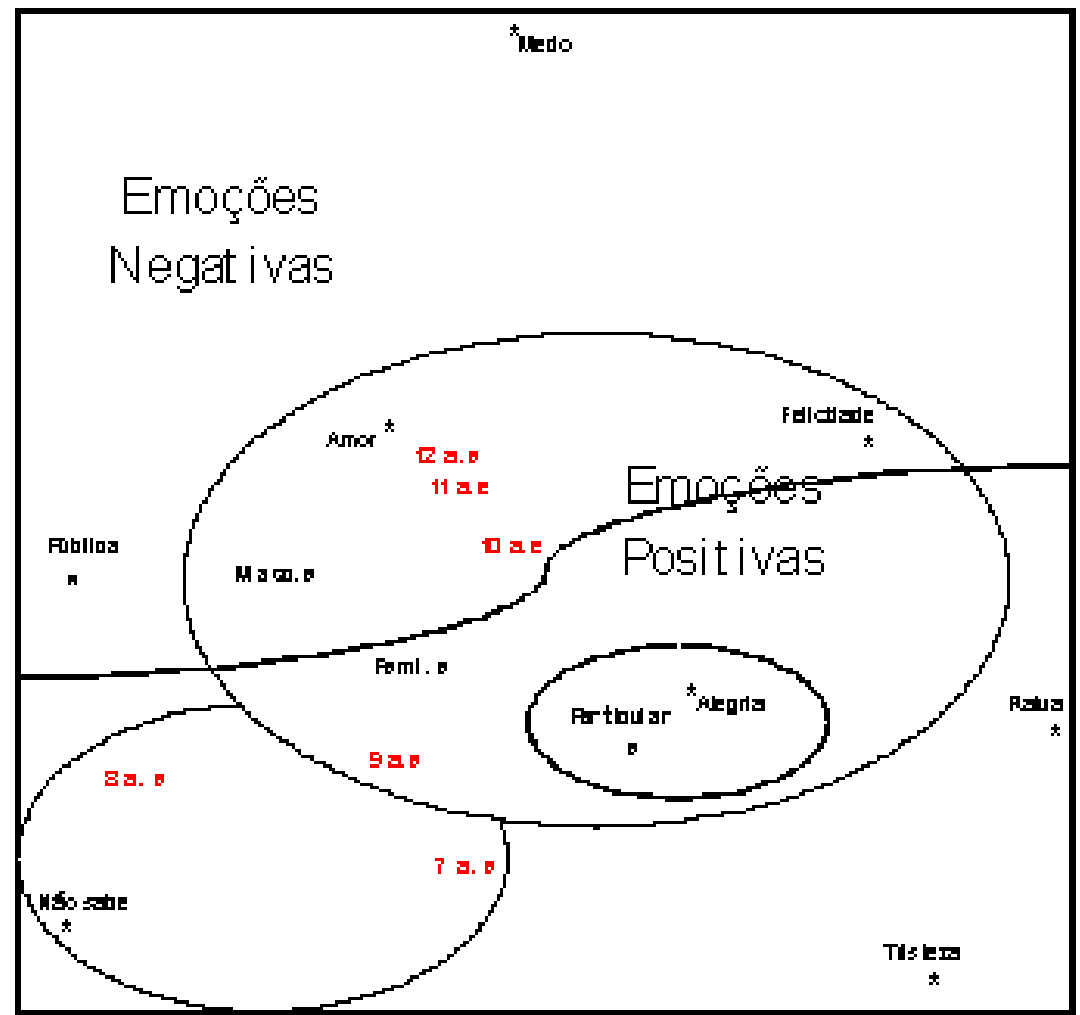

Figura 2. Análise SSA das emoções experimentadas com maior freqüência (Questão 2) considerando como variáveis externas (e) tipo de escola, sexo e faixa etária Nota. Projeção tridimensional, Coordenada 1 x 2, Coeficiente de Alienação 0,01.

menor a dificuldade de identificar as emoções que experienciam com mais freqüência (a idade explica 5,5\% da variância) $\left(\mathrm{F}_{\text {change }}(1,244) 14,24, \mathrm{p}<0,001\right)$. Isto reforça os estudos de Harris (1996) por considerar que as crianças gradualmente adquirem uma maior percepção de seus sentimentos ou de suas emoções.

\section{$3^{\text {a }}$ Questão - Conceito das Emoções}

$\mathrm{Na}$ tarefa de investigação do conceito de emoção, mediante o grande número de respostas, foi realizada uma série de categorizações. Primeiro considerando a característica das respostas, e segundo, o conteúdo das res-postas, com base na perspectiva teórica da mediação semió-tica dos processos afetivos proposta por Valsiner (2001). Para esta questão dois juízes separadamente classi-ficavam as respostas tanto em termos das características das respostas como em termos do conteúdo; a porcentagem de concordância foi de 91 e 88, respectivamente. As divergências eram dirimidas por um terceiro juiz.

No que diz respeito à característica das respostas foram construídas as seguintes categorias: definiu produzindo emoções primárias ("É sentir medo, felicidade, tristeza." - $11^{\mathrm{a}} 2 \mathrm{~m}, \mathrm{~F} .$, Part. ${ }^{4}$ ); emoções secundárias ("Pouquinho. São as amizades, é Deus." - $7^{\mathrm{a}} 10 \mathrm{~m}, \mathrm{~F}$., Part.); emoções mistas ("Alegria, tristeza, amizade,.. e amor." - $9^{\mathrm{a}} 10 \mathrm{~m}$, F., Pub.); emoções positivas ("É ficar alegre." - 7ª 9m, F., Pub.) emoções negativas ("Quando a gente fica triste... sei lá..." - $12^{\mathrm{a}} 8 \mathrm{~m}, \mathrm{~F} .$, Pub.); emo-ções positivas e negativas juntas ("Emoção é tristeza, alegria... esqueci do resto." - $9^{a} 1 \mathrm{~m}, \mathrm{~F}$., Pub.). Pelo fato de haver uma nomeação de categorias específicas de emoções, supomos que todas essas categorias e suas respectivas respostas caracterizam e exemplificam o nível lingüístico 2 do desenvolvimento ontogenético da me-diação semiótica dos processos afetivos, de acordo com a perspectiva teórica de Valsiner (2001).

Ainda quanto à característica das respostas, outras categorias foram elaboradas: definiu um conceito de emoção ("É difícil dizer... É alguma coisa que você sente quando tá sentindo alguma coisa... Né?" - $11^{\mathrm{a}} 6 \mathrm{~m}, \mathrm{M}$., Part.); não definiu (a criança diz que não sabe responder); sabia o que era emoção mas não conseguia se expressar ("Emoção é ... sei lá uma coisa ... que não sei explicar." - $9^{a} 11 \mathrm{~m}, \mathrm{~F}$., Part.). As respostas que produziram uma definição, por envolverem ações discursivas mais complexas, podem ser consideradas dentro do nível lingüístico 3 , conforme uma das respostas acima. Enquanto aquelas que sabiam o que era emoção mas não conseguiram se expressar, pode ser um indicativo de respostas no nível paralinguístico, composto por estados afetivos complexos não expressáveis por palavras.

De modo geral, entre as crianças que não produziram uma resposta, constavam aquelas que simplesmente não 
sabiam e aquelas que sabiam, mas não conseguiam se expressar. A categoria "não sabe" teve uma frequência maior nas crianças menores, o que pode indicar uma dificuldade maior de nomeação das emoções entre crianças de sete a oito anos. No entanto, convém salientar que essa categoria "não sabe" pode ser um indicador de outros fatores, como por exemplo, o não engajamento dos sujeitos no momento da entrevista. A categoria "sabe, mas não consegue explicar" ocorreu com maior incidência em crianças maiores. $\mathrm{O}$ que nos leva a pensar que com o aumento da idade as crianças atingem o nível paralinguísti-co, por estarem mais propícias a experenciarem estados afetivos cada vez mais complexos, difíceis de serem nomeados através de palavras. Tanto no nível pré-linguístico quanto no paralinguístico há ausência de palavras, mas no segundo essa ausência não ocorre por desconheci-mento e sim pelo fato do estado afetivo estar além de um enquadre semântico para criança.

Com relação ao conteúdo das respostas, foram obtidas as seguintes categorias: respostas relacionando emoção a acontecimentos positivos:

Emoção é quando, tipo assim, você conhece um homem que você sempre quis, e você fica assim, chorando de emoção, assim. É quando você conheceu tipo assim, um jogador, um jogador que você queria conhecer, e fica alegre. Quando você tem uma festa surpresa, assim, você fica muito alegre, chora. ( $9^{\mathrm{a}} 5 \mathrm{~m}, \mathrm{M}$.).

Acontecimentos negativos ("É quando briga, que acaba chorando." - $7^{\mathrm{a}} 6 \mathrm{~m}$, M., Part.), acontecimentos positivos e negativos ("Emoção é tudo o que a gente sente, seja ela ligada a um fato ruim ou um fato bom, alguma coisa que deixe a gente feliz ou não." - $10^{\mathrm{a}} 3 \mathrm{~m}, \mathrm{~F}$., Part.); relacionando a sentimentos ("Sei. É um sentimento que eu tenho, e que as pessoas têm. Que pode ser bom, ruim." - $10^{\mathrm{a}} 10 \mathrm{~m}$, M., Part.); relacionando a reação expressiva, fisiológica ("Emoção? É quando uma pessoa diz alguma coisa e a pessoa fica alegre. E... passa pela mente da pessoa que você vai sentindo um arrepio e vai começando a chorar, e a pessoa fica alegre." - $8^{\mathrm{a}} 11 \mathrm{~m}$, M., Part.); relacionando a interação social, vínculo afetivo ("É... quando a pessoa perde, assim, a mãe, o pai, e as lágrimas começam a cair. É, assim, a pessoa triste, alegre, rindo, chorando." $-9^{\mathrm{a}} 10 \mathrm{~m}$ ).

Foram computadas uma série de regressões "passo-a-passo" considerando como variáveis dependentes as categorizações na tarefa de definição da emoção e como variáveis independentes idade, sexo e tipo de escola. Constata-se que quanto maior a idade das crianças, maior uma conceitualização da emoção como um sentimento - a idade explicando $8,8 \%$ da variância $\left(\mathrm{F}_{\text {change }}(1,245)\right.$ 23,626, $\mathrm{p}<0,001)$. Nesse sentido, ao relacionar emoção com sentimento, a criança sai de um conceito reduzido ao meramente corpóreo, visível, como o choro, o sorriso e amplia sua percepção e modo de pensar contemplando a experiência mental privada de uma emoção, de acordo com a diferenciação entre emoções e sentimentos proposto por Damásio (2004). Segundo Harris (1996) as mudanças com a idade são decorrentes do reconhecimento do papel da responsabilidade social, da importância dos padrões normativos (o que pode ser expresso ou não), e o reconhecimento de um observador.

A definição da emoção como estando relacionada a sentimentos apresenta como preditor significante também o Tipo de Escola - explicando 4,7\% da variância ( $\mathrm{F}_{\text {change }}$ $(1,244) 13,366, p<0,001)$. Assim constata-se uma relação entre as crianças serem de escola particular e a produção deste conteúdo da emoção como sentimento; o que significa dizer que crianças pertencentes a esse tipo de escola apontam possuir um conhecimento sobre as emoções diferente em comparação com as crianças de escola públicas, pois consideram as peculiaridades de sentimento e emoção, de acordo com a diferenciação defendida por Damásio (2004). "Além disso, considerando os níveis linguísticos do desenvolvimento semiótico dos estados afetivos (Valsiner, 2001), o fato de terem demonstrado uma tendência maior na produção de uma definição, pode indicar a presença de uma maior elaboração situada no nível lingüístico 4, quando há ações discursivas complexas. Isto ocorre por não se restringir simplesmente a citar exemplos de emoções ou acontecimentos envolvidos por tais emoções, característicos do nível linguístico 3, quando categorias específicas de emoções são nomeadas."

Além disso, foi encontrado que quanto maior a idade, menos a criança relaciona a emoção a acontecimentos positivos e produz menos emoções positivas $-\%$ da variância explicada $1,6\left(\mathrm{~F}_{\text {change }}(1,245) 3,994, \mathrm{p}<0,047\right)$ e $4,2\left(\mathrm{~F}_{\text {change }}\right.$ $(1,245) 10,628, \mathrm{p}<0,001)$, respectivamente). Isto pode indicar que durante a trajetória percorrida pela criança em seu desenvolvimento, ocorrem mudanças emocionais, pois o conhecimento conceitual e os processos cognitivos tornam-se mais refinados, dando origem às emoções mais complexas. Deste modo, a emoção, em sua forma de expressão, não é isenta das influências da situação na qual é produzida e faz parte de um fenômeno histórico-cultural, sendo construídas, organizadas e nomeadas dentro deste contexto de inter-relações (Oliveira \& Oliveira, 1999).

Constata-se também que com o aumento da idade aumenta também a produção de respostas do tipo "Sabe, mas não sabe expressar" (\% da variância explicada foi de $\left.5,7-\mathrm{F}_{\text {change }}(1,245) 14,718, \mathrm{p}<0,001\right)$ indicando que com o avanço da idade a criança compreende a complexidade da emoção, sabe o que é mas tem dificuldade em expressar através de palavras. O que é bem diferente da resposta "Não sabe, Não sabe responder" cujo efeito preditor é indicado tanto pela idade (quanto menor a idade, maior este tipo de respostas: \% da variância explicada $2,5-\mathrm{F}_{\text {change }}(1,244)$ $6,421, \mathrm{p}<0,012$ ) como pelo tipo de escola (maior número deste tipo de respostas nas crianças de escola pública: \% da variância explicada $3,1\left(\mathrm{~F}_{\text {change }}(1,245) 7,770, \mathrm{p}<0,006\right)$.

\section{Considerações Finais}

O objetivo principal deste estudo foi investigar a organização estrutural do campo semântico conceitual da emoção em crianças em uma perspectiva êmica, visando melhor 
compreender a representação da mesma. Os resultados apontam para a existência de uma estrutura ordenada de tipo modular, tanto considerando o binômio primárias-secundárias como o binômio positivas-negativas. Este é o primeiro estudo na literatura que aponta esta estrutura ordenada.

Foi observado também que com o avanço da idade as crianças vão desenvolvendo um conceito das emoções mais elaborado, vivenciando emoções mais específicas e passando a considerar melhor as emoções negativas. Constatamos nesse estudo a relevância da variável pre-ditora idade como influenciando de forma significativa a organização estrutural do campo semântico conceitual das emoções em crianças. Em um grau menor, o tipo de escola atendido pelas crianças, pela localização e também pela distância que caracterizou as duas variáveis externas no mapa SSA, também desempenhou um papel relevante em predizer esta organização estrutural.

Nessa pesquisa não foi identificada diferença significativa na variável gênero, como também em outras pesquisas que enfocam as representações sociais das emoções (Roazzi et al., 2001), dado esse que se opõe a estudos que enfatizam como as crianças vivenciam e reagem as emoções a partir de relatos da experiência (Santos, 2006). Com base nesses estudos, supõe-se que organizar um conceito sobre emoções diferencia-se de organizar experiências emocionais quanto à relevância da variável gênero.

Convém ressaltar que, na medida em que o conhecimento acerca das emoções e as experiências pessoais trazidas pelas crianças estão interligados, observa-se a presença de processos metacognitivos, uma vez que ao refletir sobre suas emoções as crianças podem agir melhor sobre elas. Este processo contribui para o desenvolvimento e modificação do conhecimento que se tem das emoções e como este é organizado no campo semântico.

O estudo realizado constitui uma oportunidade de perceber os conceitos construídos pelas crianças, como estando organizados dentro de uma rede de significados que se relacionam e articulam-se entre si formando "teorias" que os sujeitos possuem acerca das emoções. Neste sentido, os conceitos não são vistos como entidades isoladas na mente do sujeito, mas como processos cognitivo-afetivos; processos estes que fazem parte de um todo complexo de inter-relações, no qual a história pessoal, diferentes contextos socioeconômicos, momentos históricos, modos de subjetivação, jogos de linguagem e a cultura precisam ser valorizados (Silva, 2004).

É importante contemplar a visão de que os conceitos construídos pelo ser humano, não são entidades estáveis, mas estão sempre em mudança devido à interação do sujeito com objetos de ação e conhecimento (instrumentos e signos) e com outros sujeitos com quem constrói significados coletivos (Oliveira \& Oliveira, 1999). As pessoas interagem com o ambiente, afetam e são afetadas pelo contexo social e esta interação não é apenas do cérebro ou de uma parte do corpo. Deste modo, esta pesquisa constitui um ponto de partida para outros estudos interessados em compreender como as crianças compreendem e representam suas emoções.

\section{Referências}

Abric, J.-C. (1994). L'étude expérimentale des représentations sociales. In D. Jodelet (Ed.), Les représentations sociales (4th ed., pp. 187-203). Paris: Presses Universitaires de France.

Albelson, R. P., \& Sermat, V. (1962). Multidimensional scaling of facial expressions. Journal of Experimental Psychology, 63, 546-554.

Amar, R., \& Toledano, S. (2002). HUDAP - Hebrew University Data Analysis Package. Jerusalem, Israel: Computation Center of the Hebrew University.

Averill, J. R. (1980). A constructivistic view of emotion. In R. Plutchik \& H. Kellerman (Eds.), Emotion: Theory, research, and experience: Vol. 1 (pp. 305-339). New York: Academic Press.

Barrett, L. F. (2006a). Solving the emotion paradox: Cate-gorization and the experience of emotion. Personality and Social Psychology Review, 10(1), 20-46.

Barrett, L. F. (2006b). Emotions as natural kinds? Perspectives on Psychological Science, 1, 28-58.

Bretherton, I., \& Beeghly Y. M. (1982). Talking about internal states: The acquisition of an explicit theory of mind. Developmental Psychology, 18, 906-921.

Bretherton, I., Fritz, J., Zahn-Waxler, C., \& Ridgeway, D. (1986). Learning to talk about emotions: A functionalist perspective. Child Development, 57, 529-548.

Bruner, J. (1997). Atos de significação. Porto Alegre, RS: Artes Médicas.

Camras, L. A., \& Allison, K. (1985). Children's understanding of emotional facial expressions and verbal labels. Journal of Nonverbal Behavior, 9(2), 84-94.

Canter, D. V., \& Ioannou, M. (2004). Criminals' emotional experiences during crimes. International Journal of Forensic Psychology, 1(2), 71-81.

Caron, R. F., Caron, A. J., \& Myers, R. S. (1982). 'Do infants see emotion expressions in static faces?' Child Development $56,1552-1560$.

Damásio, A. (1999). The feeling of what happens: Body and emotion in the making of consciousness. New York: Harcourt.

Damásio, A. (2004). Em busca de Espinosa: Prazer e dor na ciência dos sentimentos. São Paulo, SP: Companhia das Letras.

D'Andrade, R. G. (1995). The development of Cognitive Anthropology. Cambridge, MA: Cambridge University Press.

Davidoff, J. (2001). Language and perceptual categorization. Trends in Cognitive Science, 5, 382-387.

Davidson, R. J., Scherer, K. R., \& Goldsmith, H. H. (2003). Handbook of affective sciences (pp. 212-224). Oxford, UK: Oxford University Press.

Davies, I., \& Davidoff, J. (2000). Color categories are not universal: Replications and new evidence from a stone-age culture. Journal of Experimental Psychology: General, 129, 369-398.

Demo, P. (2007). Escola pública e escola particular: Semelhanças de dois imbróglios educacionais. Ensaio: Avaliação Políticas Públicas Educacionais, 15(55), 181-206.

Eid, M., \& Diener, E. (2001). Norms for experiencing emotions in different cultures: Inter- and intranational differences. Journal of Personality and Social Psychology, 81, 869-885.

Ekman, P. (1984). Expression and the nature of emotion. In K. S. Scherer \& P. Ekman (Eds.), Approches to emotion (pp. 
319-343). Hillsdale, NJ: Lawrence Erlbaum.

Ekman, P., \& Davidson, R. (1994). (Eds.). The nature of emotion. Fundamental questions. New York: Oxford University Press.

Ekman, P., \& Friesen, W. V. (1971). Constants across cultures in the face \& emotion. Journal of Personality \& Social Psychology, 17(2), 124-129.

Ekman, P., \& Friesen, W. V. (1978). Facial action coding system: Investigator's guide. Palo Alto, CA: Consulting Psychologist Press.

Espinosa, B. (1989). Ética (J. de Carvalho, J. F. Gomes, \& A. Simões, Trads., 4. ed.). São Paulo, SP: Nova Cultural. (Original work published 1677)

Fehr, B., \& Russell, A. J. (1984). Concept of emotion viewed from a prototype perspective. Journal of Experimental Psychology: General, 113, 464-486.

Fisher, G. A., Heise, D. R., Bohrnstedt, G. W., \& Lucke, J. F. (1985). Evidence for extending the Circumplex Model of Personality Trait Language to Self-Reported Moods. Journal of Personality and Social Psychology, 49(1), 233-242.

Harris, P. L. (1996). Criança e emoção: O desenvolvimento da compreensão psicológica. São Paulo, SP: Martins Fontes.

Izard, C. E. (1991). The Psychology of Emotions. New York: Plenum Press.

Jodelet, D. (1994). Représentations sociales: un domaine en expansion. In D. Jodelet (Ed.), Les représentations sociales ( $4^{\text {th }}$ ed., pp. 31-61). Paris: Presses Universitaires de France.

Lave, J. (1988). Cognition in practice: Mind, mathematics and culture in everyday life. Cambridge, MA: Cambridge University Press.

Lazarus, R. S. (1991). Emotion and adaptation. Oxford, UK Oxford University Press.

Levenson, R. W. (2001, October). The architecture of emotion: Form, function, and dysfunction. Paper presented at the meeting of the Society for Psychophysiological Research, Montreal, Canada.

Lindquist, K. A., Barrett, L. F., Bliss-Moreau, E., \& Russel, J. A. (2006). Language and the perception of emotion. Emotion, $6(1), 125-138$

Mandler, G. (1975). Mind and emotion. New York: Wiley.

Manstead, A. S. R. (1993). Children's representation of emotion. In C. Pratt \& A. F. Garton (Eds.), Systems of representation in children: Development and uses (pp. 185-210). New York: Wiley.

Markus, H. R., \& Kitayama, S. (1991). Culture and the self: Implications for cognition, emotion and motivation. Psychological Review, 98, 224-253.

Moscovici, S. (2000). Lo social em tiempos de transición / Diálogo com Serge Moscovici. Revista SIC (Caracas, Venezuela), 617, 302-305. Retrieved from http://www.dialogoconSergeMoscovici/SIC.html

Oliveira, M., \& Oliveira, M. (1999). Investigações cognitivas: Conceitos, linguagem e cultura. Porto Alegre, RS: Artes Médicas.

Plutchik, R. (1962). The emotions: Fact, theories \& a new model. New York: Random House.

Plutchik, R. (1994). The Psychology and Biology of Emotion. New York: Harper Collins College.

Plutchik, R., \& Conte, H. R. (1997). Circumplex Models of Personality and Emotions. Washington, DC: American Psychological Association.

Plutchik, R., \& Kellerman, H. (1980). Emotion: Theory, research, and experience: Vol. 1. New York: Academic Press.
Rieffe, C., Terwogt, M., \& Stockmann, L. (2000). Understanding atypical emotions among Children with autism. Journal of Autism and Developmental Disorders, 30, 195-203.

Roazzi, A. (1987). Pesquisa e contexto: Métodos de investigação e diferenças sócio-culturais em questão. Cadernos de Pesquisa, $62,35-44$.

Roazzi, A., \& Dias, M. G. B. B. (2001). Teoria das facetas e avaliação na pesquisa social transcultural: Explorações no estudo do juízo moral. In Conselho Regional de Psicologia - 13 ${ }^{a}$ Região: Paraíba - Rio Grande do Norte. (Ed.), A diversidade da avaliação psicológica: Considerações teóricas e práticas (pp. 157-190). João Pessoa, PB: Idéia.

Roazzi, A., Dias, M. G. B. B., Minervino, C. M., Roazzi, M., \& Pons, F. (2009). Children's comprehension of emotion: A cross-cultural investigation. In D. Elizur \& E. Yaniv (Eds.), Facet new horizons in theory construction and data analysis (pp. 83-102). Jerusalem, Israel: FTA.

Roazzi, A., Federicci, F. C. B., \& Wilson, M. (2001). A estrutura primitiva da representação social do medo. Psicologia: Reflexão e Crítica. 14(1), 57-72.

Roberson, D., \& Davidoff, J. (2000). The categorical perception of color and facial expressions: The effect of Verbal interference. Memory \& Cognition, 28, 977-986.

Rosch, E. (1975). Cognitive representations of semantic categories. Journal of Experimental Psychology: General, 104(3), 192-233.

Rosch, E., \& Lloyd, B. B. (1978). (Eds.). Cognition and categorization. Hillsdale, NJ: Lawrence Erlbaum.

Rosch, E., Varela, F., \& Thompson, E.T. (1991). The embodied mind. Cambridge, MA: MIT Press.

Russell, J. A. (1979). Affective space is bipolar. Journal of Personality and Social Psychology, 37(3), 345-356.

Russell, J. A. (1997). How shall an emotion be called? In R. Plutchik \& H. R. Conte (Eds.), Circumplex Models of Personality and Emotions (pp. 205-220). Washington, DC: American Psychological Association.

Russell, J. A., \& Widen, S. C. (2002). A label superiority effect in children's categorization of facial expressions. Social Development, 11, 30-52.

Santos, L. B. (2006). Emoções: Um estudo sobre a influência do sexo, idade e classe socioeconômica. Dissertação de Mestrado não-publicada, Programa de Pós-Graduação em Psicologia Cognitiva, Universidade Federal de Pernambuco, Recife, PE.

Silva, C. S. (2004). Afetividade e cognição: A dicotomia entre o "saber" e o "sentir" na escola. Retrieved July 16, 2007, from www.psicologia.com.pt

Schlosberg, H. (1954). Three dimensions of emotion. Psychological Review, 61, 81-88.

Souza, K. P. (2008). Crianças selvagens: A expressão das emoções após situação de extrema privação de convívio social. Dissertação de Mestrado não-publicada, Universidade Federal de Pernambuco, Recife, PE.

Valsiner, J. (2001). Comparative study of human cultural development. Madrid, España: Fundación Infancia y Aprendizaje.

Vikan, A., \& Dias, M. G. (1996). Estratégias para o controle das emoções: Um estudo transcultural entre crianças. Arquivos Brasileiros de Psicologia, 48, 80-95.

Vikan, A., Roazzi, A., \& Dias, M. G. B. B. (2009). Rating emotion communication: Display and concealment as effects of culture, gender, emotion type, and relationship. Interamerican Journal of Psychology, 43(1), 77-83.

Wagner, W. (1998). Sócio-gênese e características das repre- 
Roazzi, A., Dias, M. G. B. B., Silvia, J. O., Santos, L. B. \& Roazzi, M. M. (2011). O que é Emoção? Em Busca da Organização Estrutural do Conceito de Emoção em Crianças.

sentações sociais. In A. S. P. Moreira \& D. C. Oliveira (Eds.), Estudos interdisciplinares de representação social (pp. 3-25). Goiânia, GO: AB.

Wallon, H. (1981). Psicologia e educação da infância. Lisboa, Portugal: Estampa. (Original work published 1941)

Watson, D., \& Tellegen, A. (1985). Toward a consensual structure of mood. Psychological Bulletin, 98(2), 219-235.

Zammuner, V. L. (1998). Concepts of emotion: Emotionness, and dimensional ratings of Italian emotion words. Cognition and Emotion, 12, 243-272.

Zammuner, V. L. (2001, September). Emotion antecedents and their emotional labels in adolescents [Abstract]. In $15^{\circ}$ AIP Congress: Section of Development Psychology (pp. 232-233). Palermo, Italy. 